\title{
Verzeichnis der Abkürzungen.
}

Biblische Bücher.

$A \mathrm{~m}=$ Amos.

$1 \mathrm{Ch}=1$ Chronik.

$2 \mathrm{Ch}=2$ Chronik.

$\mathrm{Da}=$ Daniel.

Dt $=$ Deuteronomium.

Esr $=$ Esra.

Esth = Esther.

$\mathrm{Ex}=$ Exodus.

$\mathrm{E} z=$ Ezechiel.

$\mathrm{Gn}=$ Genesis.

$\mathrm{Hb}=$ Habakuk.

$\mathrm{Hg}=$ Haggai.

$\mathrm{Hi}=$ Hiob.

$\mathrm{Hl}=$ Hohestied.

$\mathrm{Ho}_{0}=$ Hosea.

Jer $=$ Jereniil.

Jes $=$ Jesaiat.

$\mathrm{J}_{\mathrm{O}}=$ Joel.

Jor $=$ Jona.

$J_{0 \mathrm{~S}}=$ Josua.

$1 \mathrm{~K}=1$ Könige.

$2 \mathrm{~K}=2$ Könige.

$\mathrm{Kl}=$ Klagelieder.

$\mathrm{Lv}=$ Leviticus.

$\mathrm{Ma}=$ Malearhi.

$\mathrm{Mi}=$ Micha.

$\mathrm{N}_{\mathrm{a}}=$ Nahum.

Neh $=$ Nehemia.

$\mathrm{Nu}=$ Numeri.

$\mathrm{Ob}=$ Obadja.

Pre = Prediger.

Ps $=$ Psalmen.

$\mathrm{Ri}=$ Richter.

$\mathrm{Ru}=$ Ruth.

$1 \mathrm{~S}=1 \mathrm{Sam}$.

$2 \mathrm{~S}=2 \mathrm{Sam}$.

Sac $=$ Sacharja.

Spr = Sprüche.

$\mathrm{Ze}=$ Zephanja.

\section{Litteratur.}

Bäd. ${ }^{2}$, Bäd. ${ }^{3}=$ Palästina u. Syrien, Handbuch f. Reisende, herausg. v. K. Bädeker. 2. Autl. Leipzig 1880. 3. Aufl. Leipzig 1891.

Barth $=J$. Barth, die Nominalbildung in den semit. Sprachen. 1. 2. Leipzig 1889. 91.

Berliner, Beitr. = Berliner, Beiträge zur hebr. Grammatik in 'T'almud und Midrasch. Berlin 1879.

Berth. = Bertheau.

Boch. = Bochart.

Bött. = F. Böttcher, ausführl. Lehrbuch der hebr. Sprache. 2 Bände. Leipzig 1866. 68.

Budde, Beitr. = Beitrïge zur Erklärung des Buches Hiob. Bonn 1876. - Urg. = Die biblische Urgeschichte untersucht. Giessen 1883.

CIS. = Corpus Inscriptionum Semiticarum ab Ac. Inscript. et Iitt. ed. Paris $1885 \mathrm{ff}$.

Corn. ${ }^{\prime}=$ Cornill.

Del. $=$ Franz Delitzsch.

Frd. Del. = Friedrich Delitzsch. heb. langu. $=$ the Hebrew Language viewed in the Light of Assyrian Research. London 1883. - Prol. $=$ Prolegomena eines neuen hebr.-aram. Wörterbuchs. Leipzig 1886. Par. $=\mathrm{W}_{0}$ lag das Paradies? Leipzig 1881.

J)erenb. = Derenbourg.

Dillm. = Dillmann.

D.L.Z. = Deutsche Litteratur\%eitung.

Ew. = Ewald.

Gesen. = Gesenius. Lehrgeb. = Gesenius, W., ausführl. gramm.- 
krit. Lehrgebäude der hebr. Sprache. Leipzig 1817.

GGA. = Göttingische Gelehrte Anzeigen. GGN. = Nachrichten v. d. K. Gesellsch. d. Wiss. etc. zu Göttingen.

Graf, gesch. Bb. = Graf, K. H., Die geschichtlichen Bücher des A. T. Leipzig 1866.

Halévy. mél. = Mélanges d'epigraphie et d'archéologie semitiques. Paris 1874.

Hitz. = Hitzig.

G. Hoffm. = Feorg Hoffmann.

Hommel, S. V. = Hommel, Fr., Die semitischen Völker u. Sprachen Bd. 1. Leipzig 1883.

$\mathrm{S} .=$ Die Namen der Säugetiere bei den südsemitischen

Hupf. $=$ Hupfeld. Völkern. Leipzig 1879.

Hw. = Handwörterbuch des Bibl. Alterthums, herausgeg. von E. A. Riehm. Bielefeld u. Leipzig $1875 \mathrm{ff}$.

.1'Th. = Jahrbücher f. deutsche Theologie.

.JPTh. = Jahrbücher für protestantische Theologie.

Joseph. = Fl. Josephus, Antiquitates.

BJ. = Bellum Judaicum.

$\mathrm{CA} .=$ Contra Apionem.

Klosterm. = Klostermann.

König = G. König, Lehrgebäude der hebr. Sprache, 1. Hälfte. Leipzig 1881.

Ktzsch. = W. Gesenius, hebr. Gramm. herausgeg. von E. Kautzsch. 25. Aufl. Leipzig 1889.

Ktzsch., ar. Gr. = E. Kautzsch, Grammatik d. Biblisch-Aramäischen. Leipzig 1884.

L. = Lucians Recension der LXX, citirt nach Librorum veteris testilmenti canonicorum pars I graece $P$. de Lagarde studio et sumpt. ed. Göttingen 1883.

Lag. = de Lagarde. Ges. Abh. = Gesammelte Abhandlungen. Leipzig 1866. - Agath = Agathangelus (Abhandl. d. Gött. Ges. d. Wiss. Bd. 35). - Erkl. $=$ Erklärung hebr. Wörter Göttingen 1880. (Ebenda Bd.

26). - Mitteil. = Mittheilun- $\mid$ REJ. = Revue des Études Juives. gen. Bd. 1. 2. 3.4. Gött. 1884 . 87. 89. 91. - Nom. = Übersicht über die im Aram., Arah. u. Hebr. übliche Bildung der Nomina (Abhandl. d. Gött. Ges. d. Wiss. Band 35). On. = Onomasticasacri. 2 Aufl. Göttingen 1887. - Psalt. Hier. = Psalterium juxta $\mathrm{He}$ braeos Hieronymi. 1874. Reg. = Register u. Nachträge zu der Übersicht. (Abhandl. d. Gött. Ges. d. Wiss. Bd. 37).-.. arm. Stud. = armenische Studien. Göttingen 1877. - Sym. $=$ Symmicta. I. II. Göttingen 1877. 80. -.-Sem. = Semitica (Abhandl. d. Gött. Ges.d.Wiss. Bd. 23). - Prov. = Anmerkungen zur griech. Übersetzung der Proverbien. Leipzig 1863. - Proph. chald. = Prophetae Chaldaice P. de Lagarde ed. Lipsiae 1872. Hag. Chald. = Hagiographa Chaldaice P. de Lagarde ed. Lipsiae 1873.

Levy $=$ J. Levy, Neuhebr. und Chald. Wörterbuch üb. d. Talmudiın. u. Midraschim. Leipzig 1876. Levy, Ch. W. = J. Levy, Chald. Wörterbuch über die Targumim. Leipzig $1867 \mathrm{flg}$.

Löw, PH. = J. Löw, aram. Pflanzennamen. Leipzig 1881.

$\mathrm{LXX}=$ alexandrinisch-griech. Ueberset $\%$ d. A. T.

Meyer, Gesch. = Ed. Meyer, Geschichte d. Alterthums. Erster Band. Stuttgart 1884.

Mich. = Michaelis. Suppl. = Supplementa ad Lexica Hebraici. Bd. 1 seq. Göttingen $1784 \mathrm{ff}$. Mordtm. u. M. = J. H. Mordtmann u. D. H. Müller, Sabäische Denkmäler. Wien 1883.

Nöld. = Nöldeke.

Ols. = J. Olshausen, Lehrbuch d. hebr. Sprache. Braunschweig 1863.

Pinsker $=$ Pinsker, S., Einleitung in dus babylonisch-hebräische Punktationssystem. Wien 1863. 


Rob. = Robinson. NBF. = Neuere Bib-
lische Forschungen. Berlin
1859. NU. = Neue Un-
tersuchungen über d. Topo-
graphie Jerusalems. Halle
1847. - Pal. = Palästina
u. die angrenzenden Länder.
Halle 1841 f. 3 Bde.

SBBA. = Sitzungsberichte der Berliner Akademie der Wissenschaften.

Schr. = Schrader. - KAT. $=$ die Keil- $a d j .=$ Adjectivum.

inschriften u. das Alte Testa- $a d v$. = Adverbium.

ment. 2. Aufl. Giessen 1883. äg. = ägyptisch.

- KG. = Keilinschriften u. a. h. = althebräisch.

Geschichtsforschung. Giessen 1878.

St. $=$ Stade, Lehrbuch der hebr. Gramm.

1. T'eil. Leipzig 1879.

Stade, Gesch. = Geschichte des Volkes Israel. Bd. 1.2. Berlin 1887.88.

St. u. Kr. = Theolog. Studien u. Kritiken.

$\mathrm{T}$. = Sixtinische Ausgabe der LXX, eitiert nach Tischendorfs Druck.

Then. = Thenius.

ThLZ. = Theologische Litteraturzeitung.

Th. St. aus Württ. = Theologische Studien aus Württemberg.

Wetzst. = Wetzstein.

Winer = G. B. Winer, Biblisches Realwörterbuch. 3. Aufl. Leipzig 1842.

Wlh. = Wellhausen. Comp. des Hex. ${ }^{2}=$

Die Composition des Hexateuchs u. der hist. Bücher. 2. Druck. Berlin 1889. - Sam. $=$ Text der BücherSam. Gött. 1871. - Prol. $^{3}=$ Prolegomena zur Geschichte Israels. 3. Aufl. Berlin 1886. - Skizzen = Skizzen und Vorarbeiten. de gent. $=$ de gentibus et familiis Judaeis quae $1 \mathrm{Ch} \mathrm{2.4}$ enumerantur. Göttingen 1870.

ZAT. = Zeitschr. f. d. a. t. Wiss.

ZDMG. = Zeitschr. d. deutsch. morgenl. Gesellschaft.

ZDPV. = Zeitschr. d. deutsch. PalästinaVereins.

Z. f. Ass. = Zeitschrift für Assyriologie. f. $=$ für. Wenn hinter einer Zahl ste-

Z. f. K. = Zeitschr. f. Keilschriftforsch.

Z. f. K. d. M. = Zeitschrift für Kunde des Morgenlandes.

ZwTh. = Zeitschr. f. wissensch. Theologie. Fam.-Nam. $\Rightarrow$ Familienname. a. L. = andere Lesart.

Appos. = Apposition.

ar. $=$ arabisch.

aram. = aramäisch.

Bed. = Bedeutung.

bed. = bedeutet, bedeuten.

bes. = besonders.

Bezeichn. = Bezeichnung.

bildl. = bildlich.

bzw. = beziehungsweise.

c. acc. $=$ mit dem Accusativ.

caus. = causativ.

coll. = collectiv.

com. $=$ commune.

concr. $=$ concret.

conj. $=$ Conjunction.

cs. = status constructus.

Dag. = Dagesch.

dah. $=$ daher.

dass. = dasselbe.

def. $=$ defectiv.

denom. = denominirt.

ders. $=$ derselbe $;$ dies. $=$ dieselbe.

d. $P .=$ der Person.

d. S. = der Sache.

Dtjes $=$ Deuterojesaia (Jes 40-66).

$d u .=$ dualis.

E. = Elohist (Ephraimäischer Erzähler).

eig. $=$ eigentlich.

ellipt. = elliptisch.

em. = emendiert, emendieren, emendiere.

en., energ. = energicus.

erkl. = erklären, erklärt.

$f$. = femininum.

hend $=$ folgende Seite, folgender Vers. $\mathrm{ff}$. $=$ folgende

Seiten, folgende Verse. 
f. d. Punkt. r. $=$ falls die Punktation $\mid$ Pent. = Pentateuch. richtig ist.

f. d. T. r. $=$ falls der Text richtig.

f. L. = falsche Lesart.

f. n. z. l. = falls nicht zu lesen.

Ggs. = Gegensatz, im Gegensatz zu.

Ggt. = Gegenteil.

Geschl. Nam. = Geschlechtsname. gewöhnl. = gewöhnlich.

Gl. = Glossem.

$H i$. $=$ Hi $\varphi^{\prime}$ il.

Hithpa. = HiOpáel.

Hithpol. = Hi@ipolel.

Ho. $=$ Ho $\varphi^{\prime}$ al.

HS. $=$ Handschrift. HSS. $=$ Handschriften.

imp. $=$ imperativus.

impf. = imperfectum.

inchoat. = inchoativ.

inf. $=$ infinitivus $=$ inf. cs.

inf. $a b s .=$ infinitivus absolutus.

insb. = insbesondere.

interj. = Interjection.

intr. = intransitiv.

J. = Jahvist (Judäischer Erzähler).

i. P. $=$ in Pausa.

Pers. $=$ Person

Pes. = Peschita.

pf. = perfectum.

$P i .=$ Pi al $^{i}$.

$p l .=$ Pluralis.

poet. = poetisch.

Präp., Präpos. = Präposition.

pt. = participium.

pt. p. = participium passivi.

$P u$. $=$ Púal.

Punkt. $=$ Punktation.

Qr. = Qerı (Qerê).

Qr. perpet. = Qers (Qere) perpetuum.

R. = Redaktor.

$S .=$ mit Suffix.

$\mathrm{s} .=$ siehe.

subst. $=$ substantivum.

scr. $d f$. $=$ scriptio defectiva.

scr. plena $=$ scriptio plena.

$s g .=$ singularis.

$s p . h .=$ späthebräisch.

st. $=$ statt.

s. u. $=$ siehe unter.

Syn. = Synonym.

jmd., jmdm., jmdn., jmds. = jemand u. s. w.

Kt. = Ke@i $\beta$.

Konj. = Konjunktion.

kult. $=$ kultisch.

l. = lies.

LA. = Lesart.

m. = mit.

$m .=$ masculinum.

Mass. $=$ Massora $;$ mass. $=$ massoretisch; MT. = Massoretiseher Text.

merkw. = merkwürdig.

Mš. = Mischna.

mś. = mischnisch.

metapl. = metaplastisch.

metonym. = metonymisch.

n. $=$ nomen.

n. gent. $=$ nomen gentilicium.

n. $p r .=$ nomen proprium.

nomin. $=$ Nominativ.

nh. $=$ neuhebräisch.

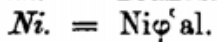

notw. $=$ notwendig.

Onk. = Onkelos.

$\boldsymbol{P}$. $=$ Pausa, in Pausa.

Pal. = Palästina.

PC. = Priestercodex.

Talm., talm. = Talmud, talmudisch.

$\mathrm{TF} .=$ Textfehler.

trans. = transitiv.

Trg. = Targum.

übtr. = übertragen.

u. d. A. = unter dem Artikel.

u. d. W. = unter dem Worte.

v. $0 .=$ u. oft; und öfters.

urspr. = ursprünglich.

v. vor Zahlen $=$ Vers.

v. = von, vom.

v. d. = von dem, von der.

Verb. $=$ Verbindung.

verk. = verkürzt.

vgl. = vergleich .

viell. $=$ vielleicht .

wahrsch. $=$ wahrscheinlich.

w. s. = wo siehe, welches siehe.

Wz. = Wurzel.

$\mathrm{z} .=\mathrm{zu}, \mathrm{zum}, \mathrm{zur}$.

Z. = Zeile.

z. l. = zu lesen.

z. St. $=$ zur Stelle.

z. str. $=\mathrm{zu}$ streichen.

z. $\mathrm{vgl}$. = zu vergleichen.

zusges. = zusammengesetr.t. 
Alle semitischen Wörter der LXX sind obne Accent aufgefüht, falls sie nicht auch griechisohe Endung haben.

Diejenigen hebräischen Formen, welche ur in Ableitungen vorkommen, sind miteinem Krois (o) bezeichnet.

Diejenigen Artikel, in welchen sämtliche Stellen des AT., worin das betr. Verbum oder Nomen vorkommt, sngeführt sind, sind am Schlusse mit einem Stern (*) bezeiohnet.

bezeichnet.

Diejecigen Worte oder Formen, wolohe uur ein mal vorkommen, sind mlteinem Kreuz (t)

( 1) Name Alef (vgl. אלכ) $\$ 26$ A. 2. Lag. Symm. I, 114. - 2) Stellung an der Spitze des Alphabets $\$ 26$ A. 3.4 , vgl. ZDMG. 26, 783. - 3) ältere Schriftformen: s. Schrifttafel bei St.; für die Siloahinschrift Ktzsch. S. 377; für sab. Denkmäler s. Mordtm. u. M. 106 . 4) Aussprache: spiritus lenis $\$ 63$ a A. Berliner, Beitr. z. hebr. Gr. 15-17. 5) Lautgesetze § 110-112. ZDMG. 8, 617. 9, 3. - 6) $\times$ als Bildungsvorsatz $\$ 255-258 .-7$ ) als Vokalzeichen $\$ 28 \mathrm{~d}$. 31. - 8) Zahlzeichen $=1$ (bei späteren Juden) $§ 26,5$.

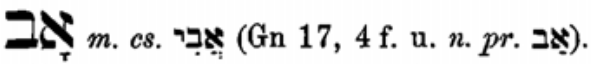

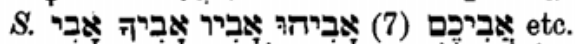

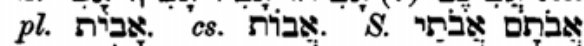
(ם $s p . h.) . ~-\$ 186.322 \mathrm{e.} 1$ 1) Vate)" (vom Großvater, Gn 28, 13), übertr. Erzeuger, Erheber, Hi 17, 14. 38, 28. - Jes 9, 5 Beutebringer (vom messian. Könige), doch s. Lag. Sem. I, 17. - Begründer einer Kunst, Lebensweise, Gn 4, 20 f.; von Jahve als Begründer des Volkes Israel, Dt 32, 6 (neben 7 TֶP). Jes 64, 7 (neben ריצ'). Ma 1, 6. 2, , 10 (neben אר: 2). 2) Vorfahr, Ahn, $1 \mathrm{~K} 15$,

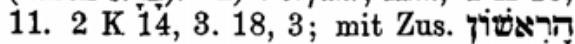
Jes 43, 27; häufig pl. Gn 47, 9. Nu 20, 15. Ps 39,13 etc.; vom Heros eponymos eines Volkes, Stammes etc., Gn 9, 18. 10, 21. 19, 37. Jes 51, 2. - 3) Beschützer, Pfleger (vgl. אם), Hi 29, 16. Jes 22, 21. V. Jahve als Beschirmer Israels, Ps 89, 27 oder seines Königs $2 \mathrm{~S}$ 7, 14. Jes 63, 16 (neben לkid). 4) Lehrer,

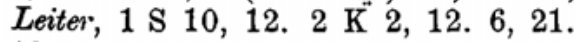
13, 14 (v. Propheten). Rj 17, 10. 18, 19 (v. Priestern als Orakelspendern); übtr. SIzGrBiEd v. STADE, Hebr. Wörterbucb. auf den Rat eines Königs, Gn 48, 8; vgl. Dillm. u. LXX zu Esth 3, 13. - Von Jahve als Israels Führer, Jer 31, 9.

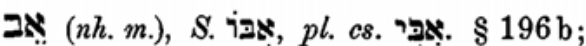
der Trieb (v. Pflanzen), Hi 8, 12. Hl 6,11*. n.pr.m. Perser, Esth 1, 10*

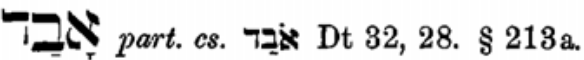

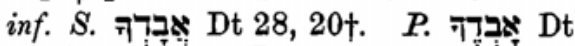

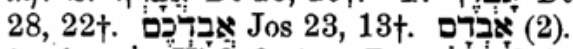

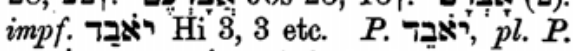

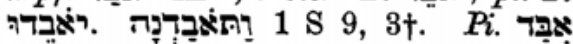
2 K̈ 21, 3. Jer 51, 55. Thr 2, 9*; impt.

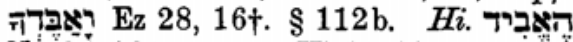

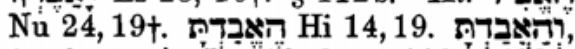
impf. אבדידָ Jer 46, 8†. §112a. 433 a. $580 \mathrm{a}$.

Qal: 1) umherirren. a) v. Tieren: sich ver. laufen, $1 \mathrm{~S} \mathrm{9,3}$. Ps 119, 176. Jer 50, 6 . das Verlaufene, Ez 34, 4. 16. b) v. Menschen: umherschweifen (v. Nomaden), Dt 26,5; hülflos umherirren, $\mathrm{Hi} 29$, 13. 31,19 ; v. den Exulanten, Jes 27, 13. c) irre gehen, c. acc. der näheren Bestimmung, דר Ps 2,12. Ew. §281 c. 2) $a b$. handen kommen, schwinden, verloren gehen, d. Besitz: Pre 5, 13. Jer 48, 36, Lebenskraft Hi 30,2, Pflanze, die verwelkt Jon 4, 10, Hoffnung Hi 8,13. Ps 9, 19 etc., Zuflucht Am 2, 14. Hi 11, 20, Weg Ps 1, 6 , Erinnerung Hi 18,17. Ps 9, 7, Weissagung, die unerfüllt bleibt $\mathrm{Ez} 12,22$, s. Smend.; Pläne, aus denen nichts wird, Ps 146, 4. - v. Personen, die man vermißt, Mi 4, 9. Sac 9, 5. - v. Völkern: ausgerottet werden, Dt 8,19 . 20; mit 7 : aus $e$. Orte verschwinden, Mi 7,2 . Hi 18 , 17; vertrieben werden, Ps 10, 16; מעֵל Dt 4, 26. Jos 23, 13. 16. - mit מִן: 